\title{
BCS Theory of Time-Reversal-Symmetric Hofstadter-Hubbard Model
}

\author{
R. O. Umucalılar ${ }^{1,2}$ and M. Iskin ${ }^{1}$ \\ ${ }^{1}$ Department of Physics, Koç University, Rumelifeneri Yolu, 34450 Sartyer, Istanbul, Turkey \\ ${ }^{2}$ Department of Physics, Mimar Sinan Fine Arts University, 34380 Şişli, Istanbul, Turkey \\ (Received 26 April 2017; revised manuscript received 28 June 2017; published 25 August 2017)
}

\begin{abstract}
The competition between the length scales associated with the periodicity of a lattice potential and the cyclotron radius of a uniform magnetic field is known to have dramatic effects on the single-particle properties of a quantum particle, e.g., the fractal spectrum is known as the Hofstadter butterfly. Having this intricate competition in mind, we consider a two-component Fermi gas on a square optical lattice with opposite synthetic magnetic fields for the components, and study its effects on the many-body BCS-pairing phenomenon. By a careful addressing of the distinct superfluid transitions from the semimetal, quantum spin-Hall insulator, or normal phases, we explore the low-temperature phase diagrams of the model, displaying lobe structures that are reminiscent of the well-known Mott-insulator transitions of the BoseHubbard model.
\end{abstract}

DOI: 10.1103/PhysRevLett.119.085301

Introduction.-The discoveries of integer and fractional quantum-Hall effects in the 1980 s brought new life to solid-state and condensed-matter physics, attracting a never-ending interdisciplinary attention since then [1]. For instance, some mathematical ideas from the topology turned out to be very successful in explaining the robustness of these effects, making the so-called topological insulators a very popular research theme in modern physics. These materials are intrinsically insulating in the bulk but have conducting edge or surface states that are robust against local perturbations [2]. While the earlier proposals require a broken timereversal $(\mathcal{T})$ symmetry as can be realized under an external magnetic field, the quantum spin-Hall insulators (SHI) preserved it $[3,4]$ in such a way that the currents carried by electrons with different spin states flow in opposite directions along the edges of the sample without dissipation.

In the mean time, the successful creation of atomic BECs in the 1990s and the tunable BCS-BEC crossover in the 2000s ignited researchers to transfer many of the model Hamiltonians developed in physics all across the board into the realm of ultracold atomic systems [5]. For instance, the recent production of synthetic magnetic fields for neutral atoms [6] was followed by the realization of the celebrated Harper-Hofstadter model $[7,8]$ in the presence of an optical lattice [9-15], the $\mathcal{T}$-preserving schemes of which were also developed to realize the quantum spin-Hall Hamiltonians [9,11]. Besides having an intriguing fractal spectrum, the generic Harper-Hofstadter model not only features the integer quantum-Hall effect [16] but it also hosts Dirac-cone physics for certain magnetic fluxes. These cones are at the heart of certain phenomena in graphenetype materials with a honeycomb lattice, including the quantum spin-Hall effect with an additional gap-opening mechanism [17] and the semimetal (SM)-BCS superconductivity transition $[18,19]$.
The Hofstadter physics is enriched even more by the effects of interactions. For instance, while it gives rise to a complicated shape for the transition boundary between the superfluid (SF) and Mott-insulator phases in the context of the repulsive Bose-Hubbard model [20,21], it promotes a playground for a variety of inhomogeneous SF phases with vortex-lattice or stripe orders in the context of the attractive Hubbard model [22-24]. Furthermore, the $\mathcal{T}$-preserving Hofstadter-Hubbard models have also been investigated soon after its realizations with cold atoms [9,11], offering a wealth of phase transitions. For instance, while the repulsive interactions may pair up the fractional quantum-Hall states to form fractional quantum spin-Hall states [25] in a Bose gas, it may drive a phase transition from a SM to an antiferromagnetic insulator in a Fermi gas [26]. In addition, the attractive interactions in a Fermi gas may drive phase transitions from a SM or a SHI to a SF phase [27-29], motivating this work.

In this Letter, we present a systematic analysis of the $\mathrm{SF}$ transition in a $\mathcal{T}$-symmetric Hofstadter-Hubbard model, and explore the resultant phase diagrams for a wide range of model parameters. Unlike the existing literature on the Hofstadter-Hubbard model with a broken $\mathcal{T}$-symmetry [22-24], we first show that the SF phase is characterized by a spatially uniform order parameter, despite the presence of a complicated single-particle spectrum brought about by the interplay of the lattice potential and the magnetic field. Based on this observation, we identify distinct transitions from the SM, SHI, normal $(N)$, and vacuum $(V)$ phases to the homogenous SF phase with respect to the singleparticle density of states of the multiband energy spectrum. See Fig. 1 for a typical illustration, whose lobe structures are reminiscent of the Mott-insulator transitions of the SF Bose gas on a lattice. We also pay special attention to the magnetic-flux dependence of the interaction threshold for 

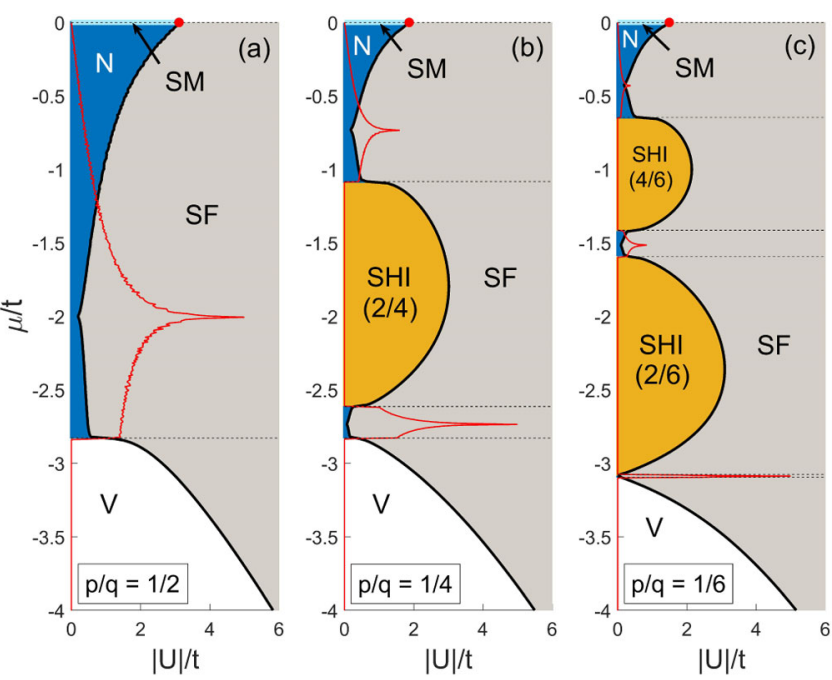

FIG. 1. Phase diagrams for $\alpha=p / q$ with $p=1$ and even- $q$ denominators (a) $q=2$, (b) $q=4$, and (c) $q=6$. Critical interaction strength $\left|U_{c}\right| / t$ is shown as a function of $\mu / t$ by a thick black curve separating $V$ (particle vacuum), $N$ (normal), SM (semimetal), quantum SHI (spin-Hall insulator), and SF (superfluid) phases at $k_{B} T=10^{-4} t$. Total filling $F$ of an SHI phase is shown in parentheses. Red dot at $\mu=0$ shows the triple point at which the $N, \mathrm{SM}$, and SF phases meet. Density of states $D(\varepsilon)$ is displayed in arbitrary units by a thin red curve ( $\varepsilon$ and $\mu$ axes coincide). Horizontal dashed lines mark the band edges including $\varepsilon=0$.

the SM-SF triple point and determine the hallmark attributes of the SF order parameter depending on the type of the transition, providing analytical expressions in various limits. Besides capturing the essential physics of the model Hamiltonian, we hope that our simpler mean-field results may also work as a benchmark for more accurate but numerically demanding QMC simulations [27].

Mean-field theory.-To describe the kinematics of a quantum particle in a tight-binding square lattice potential, we start with the following single-particle Hamiltonian, $H_{B}=-t \sum_{\langle i j\rangle \sigma}\left(e^{i 2 \pi \phi_{i j}^{\sigma}} c_{i \sigma}^{\dagger} c_{j \sigma}+\right.$ H.c. $)$ where $t>0$ is the hopping amplitude between nearest-neighbor sites $\langle i j\rangle$; i.e., $c_{i \sigma}^{\dagger}\left(c_{i \sigma}\right)$ creates (annihilates) a fermion at site $i \equiv$ $\left(i_{x}, i_{y}\right)$ with pseudospin index $\sigma \equiv\{\uparrow, \downarrow\}$, and H.c. is the Hermitian conjugate. The perpendicular magnetic field is taken into account via minimal coupling, where the hopping particle acquires a spin-dependent phase, $\phi_{i j}^{\sigma}=$ $\left(s_{\sigma} / \phi_{0}\right) \int_{\mathbf{r}_{j}}^{\mathbf{r}_{i}} \mathbf{A} \cdot d \mathbf{r}$, with $s_{\uparrow}=+1$ and $s_{\downarrow}=-1$. Here, $\phi_{0}$ is the magnetic-flux quantum and $\mathbf{A}=(0, B x)$ is the vector potential in the Landau gauge with $B$ the magnitude of the effective magnetic field. For a given magnetic-flux quanta per unit cell $\alpha=B a^{2} / \phi_{0}$, with $a \rightarrow 1$ the lattice constant, the particle gains an Aharonov-Bohm factor $\exp \left(\mathrm{i} 2 \pi s_{\sigma} \alpha\right)$ after traversing a loop around the unit cell. This is the socalled time-reversal-symmetric Hofstadter model [26,27] as realized in recent cold-atom experiments [9,11]. When $\alpha$ is a rational fraction $p / q$ with $p$ and $q$ relatively prime integers, the spectrum for each spin state consists of $q$ subbands that split from the tight-binding $s$ band of the field-free case. Energy versus $\alpha$ diagram has a fractal structure and is usually called the Hofstadter butterfly [8].

Under the validity of this model, the noninteracting Fermi gas is described by $H_{0}=H_{B}-\mu \sum_{i \sigma} n_{i \sigma}$, where $n_{i \sigma}=c_{i \sigma}^{\dagger} c_{i \sigma}$ is the number operator and $\mu$ is the common chemical potential for both spin states. Furthermore, having short-ranged attractive interactions in mind, we adopt a BCS-like mean-field approximation for pairing, and consider an on-site term, $H_{I}=-\sum_{i}\left(\Delta_{i} c_{i \uparrow}^{\dagger} c_{i \downarrow}^{\dagger}+\right.$ H.c. $)-$ $\sum_{i}\left|\Delta_{i}\right|^{2} / U$, where $\Delta_{i}=U\left\langle c_{i \uparrow} c_{i \downarrow}\right\rangle$ is the SF order parameter. Here, $U \leq 0$ and $\langle\ldots\rangle$ denotes the thermal average. Next, we switch to the momentum-space representation, and define $n=1, \ldots, q$ band operators $d_{\mathbf{k} n \sigma}$ in terms of the Fourier-expansion coefficients of $c_{i \sigma}$, i.e., $c_{\mathbf{k} \beta \sigma}=$ $\sum_{n} g_{\beta \sigma}^{n}(\mathbf{k}) d_{\mathbf{k} n \sigma}$, where $g_{\beta \sigma}^{n}(\mathbf{k})$ is the $\beta$ th component of the $n$th eigenvector of the single-particle problem with energy $\varepsilon_{\mathbf{k} n \sigma}$. Since the magnetic field imposes a new translational symmetry and enlarges the unit cell by a factor of $q$ in the $x$ direction, the Brillouin zone is reduced to $\mathbf{k}_{x} \in[-\pi / q, \pi / q)$ and $\mathbf{k}_{y} \in[-\pi, \pi)[8,30]$, and we label inequivalent sites in the enlarged cell by $\beta=0, \ldots, q-1$, where $i_{x}=s q+\beta$ with $s$ locating the supercell. The total k-space Hamiltonian $H_{M F}=H_{0}+H_{I}$ can be written as

$$
\begin{aligned}
H_{M F}= & \sum_{n \mathbf{k} \sigma} \epsilon_{\mathbf{k} n \sigma} d_{\mathbf{k} n \sigma}^{\dagger} d_{\mathbf{k} n \sigma}-\frac{M}{q U} \sum_{l \beta}\left|\Delta_{\beta}^{l}\right|^{2} \\
& -\sum_{l \beta n n^{\prime} \mathbf{k}}\left[\Delta_{\beta}^{l} g_{\beta \uparrow}^{n *}\left(\mathbf{k}_{+}^{l}\right) g_{\beta \downarrow}^{n^{\prime} *}\left(\mathbf{k}_{-}^{l}\right) d_{\mathbf{k}_{+}^{l} n \uparrow}^{\dagger} d_{\mathbf{k}_{-}^{l} n^{\prime} \downarrow}^{\dagger}+\text { H.c. }\right],
\end{aligned}
$$

where $\epsilon_{\mathbf{k} n \sigma}=\varepsilon_{\mathbf{k} n \sigma}-\mu$, and the $q \times q$ order parameter set $\quad \Delta_{\beta}^{l}=-(q U / M) \sum_{n n^{\prime} \mathbf{k}} g_{\beta \uparrow}^{n}\left(\mathbf{k}_{+}^{l}\right) g_{\beta \downarrow}^{n^{\prime}}\left(\mathbf{k}_{-}^{l}\right)\left\langle d_{\mathbf{k}_{-}^{l} n^{\prime} \downarrow} d_{\mathbf{k}_{+}^{l} n \uparrow}\right\rangle$ determines $\Delta_{i}$ through $\Delta_{i}=\sum_{l} \Delta_{\beta}^{l} e^{\mathrm{i}\left(Q_{l x} s+Q_{l y} i_{y}\right)}$. Here, $M$ is the total number of lattice sites and $\mathbf{k}_{ \pm}^{l}= \pm \mathbf{k}+\mathbf{Q}_{l} / 2$ with $\mathbf{Q}_{l}=\left(Q_{l x}, Q_{l y}\right)$ the center-of-mass momentum of Cooper pairs. As $\varepsilon_{\mathbf{k} n \sigma}$ is $q$-fold degenerate in any given band for momenta $\mathbf{k}$ and $\mathbf{k}+\mathbf{K}_{l}$ with $\mathbf{K}_{l} \equiv\{(0,2 \pi l p / q)\}$ and $l=0, \ldots, q-1$, we treat pairing with $\mathbf{Q}_{l} \equiv \mathbf{K}_{l}$ on equal footing [22].

By solving these self-consistency equations for a wide range of parameters, we confirm that the thermodynamic potential is minimized by the solution $\Delta_{\beta}^{l}=\Delta \delta_{l 0}$ with $\delta_{i j}$ the Kronecker delta, in such a way that a single order parameter $\quad \Delta=-(U / M) \sum_{n \mathbf{k}}\left\langle d_{\mathbf{k} n \downarrow} d_{-\mathbf{k} n \uparrow}\right\rangle$ characterizes the resultant homogenous SF phase [31]. This is unlike the usual Hofstadter-Hubbard model with a broken $\mathcal{T}$ symmetry, where inhomogenous SF phases require a nontrivial set of $q \times q$ parameters, e.g., a vortex-lattice solution $[22,24]$. The large $|U| / t$ limit is particularly illuminating beyond which the entire Fermi gas consists of many-body 
bound states that eventually form two-body bound states, i.e., bosonic molecules, experiencing no net magnetic field. Thus, thanks to the $\mathcal{T}$ symmetry of the present model, the order parameter equation simplifies to

$$
\frac{1}{U}=-\frac{1}{M} \sum_{n \mathbf{k}} \frac{1}{2 E_{\mathbf{k} n}} \tanh \left(\frac{E_{\mathbf{k} n}}{2 k_{B} T}\right),
$$

where $E_{\mathbf{k} n}=\sqrt{\epsilon_{\mathbf{k} n}^{2}+\Delta^{2}}$ is the quasiparticle energy in a given band $n$ as the dispersion $\varepsilon_{\mathbf{k} n}$ is equal for both spin states, $\Delta$ is taken as a real parameter without loosing generality, $k_{B}$ is the Boltzmann constant, and $T$ is the temperature. Here, $\mu$ is determined by the total number of particles $N=\sum_{i \sigma}\left\langle n_{i \sigma}\right\rangle$, leading to the number equation

$$
F=\frac{1}{q M} \sum_{n \mathbf{k}}\left[1-\frac{\epsilon_{\mathbf{k} n}}{E_{\mathbf{k} n}} \tanh \left(\frac{E_{\mathbf{k} n}}{2 k_{B} T}\right)\right],
$$

where $0 \leq F=N / M \leq 2$ is the total particle filling. While we recover the familiar expressions $\Delta=(|U| / 2-$ $\left.4 t^{2} /|U|\right) \sqrt{F(2-F)}$ and $\mu=-\left(|U| / 2-8 t^{2} /|U|\right)(1-F)$ for the bosonic molecules in the strong-coupling limit when $\Delta \gg t$ or equivalently $|U| \gg t$, the weak-coupling limit turns out to be quite rich, showing a variety of distinct phases and transitions in between. Next, we construct typical phase diagrams for $\mu \leq 0$, as the solutions are mirror-symmetric around $\mu=0$ or, equivalently, $F=1$ due to the particle-hole symmetry of the parent Hamiltonian.

Low-temperature phase diagrams.-The critical interaction threshold $\left|U_{c}\right|$ above which the system develops SF correlations with $\Delta \neq 0$ can be obtained by setting $\Delta \rightarrow 0$ in Eqs. (2) and (3). For instance, our low- $T$ phase diagrams that are shown in Figs. 1 and 2 reveal four distinct SF transitions: (i) a SM-SF transition when $\mu=0$ or $F=1$ for even $q$, (ii) a SHI-SF transition when $\mu$ lies within a band gap or $F=2 s / q$ with $s \leq q$ an integer, (iii) a $N$-SF
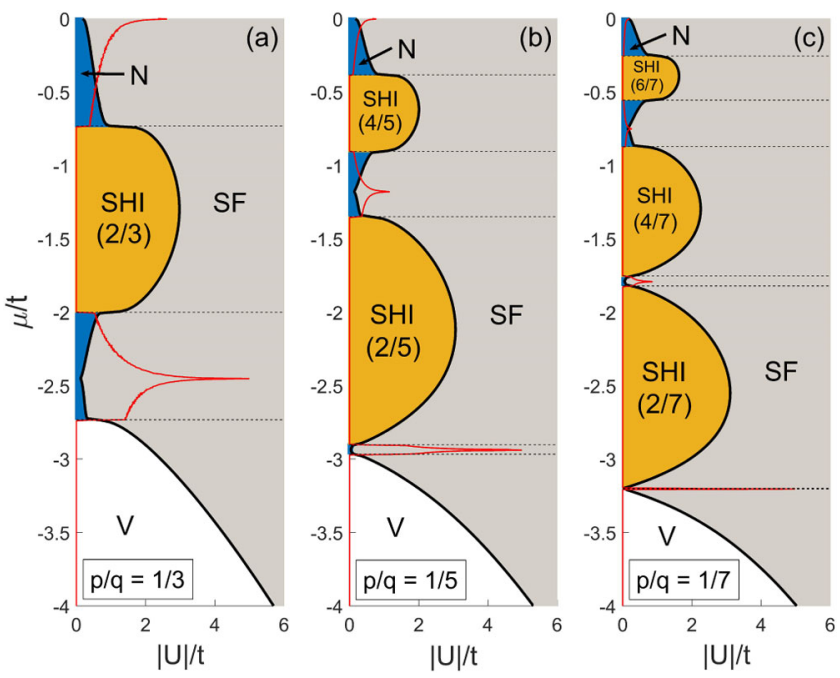

FIG. 2. Similar to Fig. 1 but for odd- $q$ denominators (a) $q=3$, (b) $q=5$, and (c) $q=7$. transition when $\mu$ lies within a band or $F \neq 2 s / q$, and (iv) a $V$-SF transition when $\mu$ lies below the lowest available band or $F \rightarrow 0$. We note that even though the mean-field framework is known to be less accurate in two dimensions, not to mention the critical role played by the multiband spectrum, it should be considered as a qualitative description of the system at the best-case scenario. Furthermore, given its semianalytic nature, it not only helps us build the intuition behind these competing phases but it also serves as an ultimate benchmark for fully numerical QMC simulations [27].

First of all, the SM-SF transition at $F=1$ (i.e., halffilling) for even- $q$ denominators is caused by the presence of $q$ linearly dispersing Dirac cones in the magnetic Brillouin zone. It turns out that even though $\Delta \rightarrow 0$ at the transition boundary, the Dirac cones guarantee a small energy window of the $\mathbf{k}$-space region around $\varepsilon=0$ with $\Delta>|\varepsilon|$, no matter how small the energy window is. This leads to a finite triple point $U_{c} \neq 0$ as shown in Fig. 1. The $T=0$ limit of $U_{c}$ is determined by $M / U_{c}=$ $-\sum_{n \mathbf{k}} 1 /\left(2\left|\varepsilon_{\mathbf{k} n}\right|\right)$, near which $\Delta_{0}=\left(U_{c}-U\right) /\left(C U_{c}^{2}\right)$ increases linearly with $|U|$, where $D(\varepsilon)=C|\varepsilon|$ is the low-energy density of states near the cones. At $T=0$, we find $\left|U_{c}\right| / t \approx 3.111$ and 1.871 for $q=2$ and 4 , respectively, and the complicated dependence of $U_{c}$ on $q$ is closely related to the bandwidth of the central bands as shown in Fig. 3(a) for $q$ up to 100. For a given prime number $p$, the oscillatory dependence shown in Fig. 3(b) is a consequence of the self-similar fractal spectrum in such a

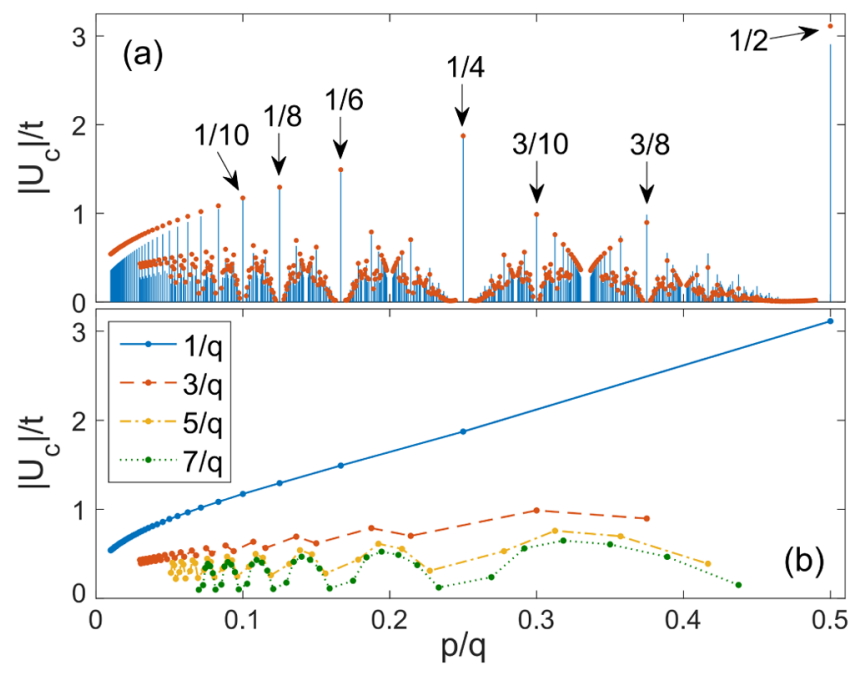

FIG. 3. SM-SF transition thresholds at $k_{B} T=10^{-4} t$ for all unique $\alpha=p / q \leq 1 / 2$ ratios with even- $q$ denominators up to 100. (a) While the red points are our self-consistent solutions, the vertical blue lines correspond to $(2 W / t)^{0.44}$, where $W$ is the total bandwidth of the 2 central bands for a given $\alpha$. In (b) the same data are grouped according to the numerator $p$, where the connecting lines are drawn as a guide, showing the oscillatory dependence. 
way that each $\alpha$ interval between $1 / q$ and $1 /(q+2)$ contains $p-1$ data points with distinct $p / q$ ratios.

Away from half filling, when the filling fraction is $F=$ $2 s / q$ with $s \leq q$ an integer, there are $s$ fully occupied bands and the system is a SHI. At $T=0$, the SHI-SF transition boundary is determined by $M / U_{c}=-\sum_{n \mathbf{k}} 1 /\left(2\left|\epsilon_{\mathbf{k} n}\right|\right)$, near which $\Delta_{0}=\sqrt{\left(U_{c}-U\right) /\left(C_{0} U_{c}^{2}\right)}$ increases as a squareroot with $|U|$, where $M C_{0}=\sum_{n \mathbf{k}} 1 /\left(4\left|\epsilon_{\mathbf{k} n}\right|^{3}\right)$ is a constant for a given $q$. On the other hand, since $F \neq 2 s / q$ corresponds to a $N$ phase with a partially occupied band, we find that $U_{c} \rightarrow 0$ as $T \rightarrow 0$ [32], near which $\Delta_{0}=$ $2|\mu| \exp \{1 /[D(\mu) U]-1\}$ increases exponentially with $|U|$ for even- $q$ values close to the half filling when $\Delta_{0} \ll|\mu| \approx 0$. The BCS-like $D(\varepsilon)$ dependence is clearly seen in Figs. 1 and 2, where, while $T \neq 0$ causes $U_{c} \neq 0$ in general, its magnitude is inversely related to $D(\varepsilon)$. Note that, since the total band width is constrained by $8 t$ in the $q \rightarrow \infty$ limit, increasing $q$ flattens the band widths of each of the $q$ bands, leading to a singular $D(\varepsilon)$ with discrete structure. As the $N$ regions shrink and become hardly visible even at $T \neq 0$, our large- $q$ phase diagrams [32] are reminiscent of the Mott-insulator transitions of the SF Bose gas on a lattice $[5,33]$.

This low- $T$ analysis clearly show that, depending on whether $\Delta_{0}$ increases as a linear, square-root, or exponential function of $|U|$, one can distinctly characterize the corresponding type of the SF transition. For instance, we illustrate $\Delta$ for all 3 types on the right axis of Fig. 4 , where we set $\alpha=1 / 4$ and $k_{B} T=10^{-4} t$. To further support this finding, we also show the ratio $k_{B} T_{c} / \Delta$ on the left axis of the same figure, where $T_{c}$ is the corresponding SF transition temperature. Setting $\Delta=0$ and $\mu=0$ in Eq. (2),
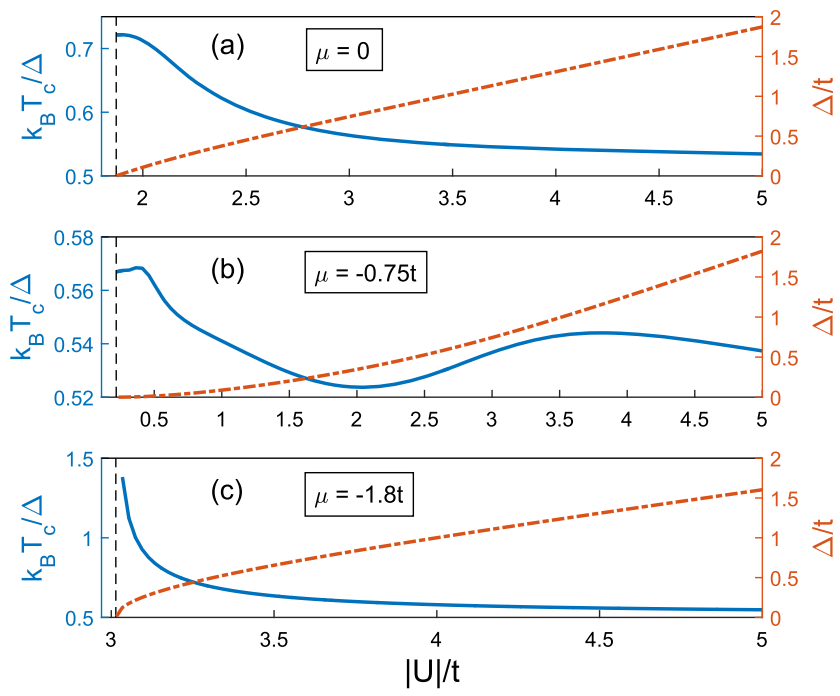

FIG. 4. (Left) Ratios of the critical temperature $k_{B} T_{c}$ and $\Delta$ at $k_{B} T=10^{-4} t$ for $\alpha=1 / 4$. (Right) $\Delta / t$ as dash-dotted red lines. Vertical dashed lines correspond to the $\left|U_{c}\right| / t$ thresholds for the (a) SM-SF, (b) $N$-SF, and (c) SHI-SF transitions discussed in the text. and assuming $T_{c}$ is small, we find $k_{B} T_{c}=$ $\left(U_{c}-U\right) /\left(2 \ln 2 C U_{c}^{2}\right)$ as $U \rightarrow U_{c}$ in the weak-coupling limit near the $T=0$ SM-SF transition boundary. This suggests $k_{B} T_{c} / \Delta_{0}=1 /(2 \ln 2) \approx 0.721$ in the $U \rightarrow U_{c}$ limit, which is in perfect agreement with Fig. 4(a). Similarly, setting $\Delta=0$ and $\mu \approx 0$ in Eq. (2), and assuming $k_{B} T_{c} \ll|\mu|$, we find $k_{B} T_{c}=(2|\mu| / \pi) \exp \{1 /[D(\mu) U]+$ $\gamma-1\}$ for even- $q$ values as $U \rightarrow 0$ in the weak-coupling limit near the $N$-SF transition boundary, where $\gamma \approx 0.577$ is the Euler's constant. This suggests $k_{B} T_{c} / \Delta_{0}=e^{\gamma} / \pi \approx$ 0.567 in the $U \rightarrow U_{c}=0$ limit, which is again in perfect agreement with Fig. 4(b). Last, setting $\Delta=0$ in Eq. (2), and assuming $\mu$ is in the middle of one of the band gaps and $k_{B} T_{c} \ll A$ is small, we find $k_{B} T_{c} \sim$ $-A /\left\{2 \ln \left[A q\left(U_{c}-U\right) /\left(4 U_{c}^{2}\right)\right]\right\}$ as $U \rightarrow U_{c}$ in the weakcoupling limit near the $T=0$ SHI-SF transition boundary, where $A$ is approximately the corresponding band gap between the highest-occupied and lowest-unoccupied band. This suggests $k_{B} T_{c} / \Delta_{0} \rightarrow \infty$ in the $U \rightarrow U_{c}$ limit, i.e., a logarithmic divergence of the form $\lim _{x \rightarrow 0} 1 /|\sqrt{x} \ln x|$, which is also in perfect agreement with Fig. 4(c). In the strong-coupling limit when $|U| \gg t$, it is well known that the mean-field $T_{c}$ is solely related to the formation of pairs and it has nothing to do with the actual SF transition. Setting $\Delta=0$ in Eq. (2), and assuming $k_{B} T_{c} \gg|\mu|$ leads to $k_{B} T_{c}=|U| / 4$ which is the case around $\mu \approx 0$ (or half filling $F \approx 1$ ), while assuming $k_{B} T_{c} \ll|\mu|$ leads to $k_{B} T_{c}=$ $-|\mu| / \ln (F / 2)$ and $|\mu| \approx|U| / 2$, which is the case for low filling $F \ll 1$. Therefore, in the $|U| \gg t$ limit, while all 3 of our numerical results approach $k_{B} T_{c} / \Delta_{0} \rightarrow 0.5$ in Fig. 4, we expect a diverging $k_{B} T_{c} / \Delta_{0} \simeq-1 /[\sqrt{2 F} \ln (F / 2)]$ ratio as $F \rightarrow 0$. It is notable that this logarithmic divergence is quite similar in structure to that of the weak-coupling one near the SHI-SF transition discussed just above.

Furthermore, in the weak-coupling limit near the SF transition boundary, using $\Delta \ll k_{B} T_{c}$ near $T_{c}$, we find $\Delta=\sqrt{8 \ln 2} k_{B} T_{c} \sqrt{1-T / T_{c}} \approx 1.698 \Delta_{0} \sqrt{1-T / T_{c}}$ for even- $q$ values when $\mu=0$ is at half filling, $\Delta=$ $\sqrt{8 \pi^{2} /[7 \zeta(3)]} k_{B} T_{c} \sqrt{1-T / T_{c}} \approx 1.736 \Delta_{0} \sqrt{1-T / T_{c}}$ for even- $q$ values when $\mu \approx 0$ is in the band, and $\Delta=\left\{\left[1 /\left(M C_{0} k_{B} T_{c}\right)\right] \sum_{n \mathbf{k}} e^{-\left|\epsilon_{\mathbf{k} n}\right| /\left(k_{B} T_{c}\right)}\right\}^{1 / 2} \sqrt{1-T / T_{c}} \sim$ $\sqrt{A /\left(2 k_{B} T_{c}\right)} \Delta_{0} \sqrt{1-T / T_{c}}$ when $\mu$ is in the band gap with $A \gg k_{B} T_{c}$. Lastly, in the large- $q$ limit when $\left|\epsilon_{\mathbf{k} n}\right| \ll$ $k_{B} T_{c}$ for any one of the bands, we find $\Delta=\sqrt{12} k_{B} T_{c} \sqrt{1-T / T_{c}}=\sqrt{3} \Delta_{0} \sqrt{1-T / T_{c}}$, the coefficient $\sqrt{3} \approx 1.732$ of which almost coincides with that of the BCS expression. Therefore, the $T$ dependences of $\Delta$ are all alike near $T_{c}$ up to the prefactor, in the characteristic form of a second-order phase transition.

We end this Letter by noting that, in addition to the recent proposals for distinguishing different SHI lobes $[11,27]$, the SM-SF and SHI-SF transitions may be directly probed by measuring the density profiles, and studying the 
resultant "wedding cake" structures [5]. In addition, the SHI phases can be further identified by the density profiles via an effective "Hall conductance" introduced through the well-known Streda formula [34].

Conclusions. - In summary, we used the $\mathcal{T}$-symmetric Hofstadter-Hubbard model on a square optical lattice in order to describe and study the BCS-pairing correlations of a two-component Fermi gas that is experiencing opposite synthetic magnetic fields for its components. We found rich phase diagrams involving distinct SF transitions from the $\mathrm{SM}$, quantum SHI, or $N$ phases, the lobe structures of which are reminiscent of the Mott-insulator transitions of the SF Bose gas on a lattice $[5,33]$. Given the ongoing coldatom experiments in simulating such models $[9,11]$, there is no doubt that even though our mean-field description may only capture the qualitative physics of the model Hamiltonian in two dimensions, which is further complicated by the multiband spectrum, it not only offers a lessaccurate, but analytically tractable analysis in helping us shape the intuition behind the competing phases, but it also paves the way as an ultimate benchmark for more accurate yet fully numerical QMC simulations [27].

M. I. acknowledges funding from TÜBİTAK Grant No. 1001-114F232 and the BAGEP award of the Turkish Science Academy.

[1] K. v. Klitzing, G. Dorda, and M. Pepper, New Method for High-Accuracy Determination of the Fine-Structure Constant Based on Quantized Hall Resistance, Phys. Rev. Lett. 45, 494 (1980); D. C. Tsui, H. L. Stormer, and A.C. Gossard, Two-Dimensional Magnetotransport in the Extreme Quantum Limit, Phys. Rev. Lett. 48, 1559 (1982); D. Yoshioka, The Quantum Hall Effect (Springer-Verlag, Berlin, 2002).

[2] M. Z. Hasan and C. L. Kane, Colloquium: Topological insulators, Rev. Mod. Phys. 82, 3045 (2010).

[3] C. L. Kane and E. J. Mele, $Z_{2}$ Topological Order and the Quantum Spin Hall Effect, Phys. Rev. Lett. 95, 146802 (2005).

[4] B. A. Bernevig, T. L. Hughes, and S.-C. Zhang, Quantum spin hall effect and topological phase transition in $\mathrm{HgTe}$ quantum wells, Science 314, 1757 (2006); M. König, S. Wiedmann, C. Brüne, A. Roth, H. Buhmann, L. W. Molenkamp, X.-L. Qi, and S.-C. Zhang, Quantum Spin Hall Insulator State in HgTe Quantum Wells, Science 318, 766 (2007).

[5] M. Lewenstein, A. Sanpera, V. Ahufinger, B. Damski, A. Sen De, and U. Sen, Ultracold atomic gases in optical lattices: mimicking condensed matter physics and beyond, Adv. Phys. 56, 243 (2007); I. Bloch, J. Dalibard, and W. Zwerger, Many-body physics with ultracold gases, Rev. Mod. Phys. 80, 885 (2008).

[6] J. Dalibard, F. Gerbier, G. Juzeliūnas, and P. Öhberg, Colloquium: Artificial gauge potentials for neutral atoms, Rev. Mod. Phys. 83, 1523 (2011); N. Goldman, G. Juzeliūnas, P. Öhberg, and I. B. Spielman, Light-induced gauge fields for ultracold atoms, Rep. Prog. Phys. 77, 126401 (2014).

[7] P. G. Harper, Single band motion of conduction electrons in a uniform magnetic field, Proc. Phys. Soc. London Sect. A 68, 874 (1955)

[8] D. R. Hofstadter, Energy levels and wave functions of Bloch electrons in rational and irrational magnetic fields, Phys. Rev. B 14, 2239 (1976).

[9] M. Aidelsburger, M. Atala, M. Lohse, J. T. Barreiro, B. Paredes, and I. Bloch, Realization of the Hofstadter Hamiltonian with Ultracold Atoms in Optical Lattices, Phys. Rev. Lett. 111, 185301 (2013).

[10] H. Miyake, G. A. Siviloglou, C. J. Kennedy, W. C. Burton, and W. Ketterle, Realizing the Harper Hamiltonian with Laser-Assisted Tunneling in Optical Lattices, Phys. Rev. Lett. 111, 185302 (2013).

[11] C. J. Kennedy, G. A. Siviloglou, H. Miyake, W. C. Burton, and Wolfgang Ketterle, Spin-Orbit Coupling and Spin Hall Effect for Neutral Atoms without Spin-Flips, Phys. Rev. Lett. 111, 225301 (2013).

[12] C. J. Kennedy, W. C. Burton, W. C. Chung, and W. Ketterle, Observation of Bose-Einstein condensation in a strong synthetic magnetic field, Nat. Phys. 11, 859 (2015).

[13] M. Aidelsburger, M. Lohse, C. Schweizer, M. Atala, J. T. Barreiro, S. Nascimbene, N. R. Cooper, I. Bloch, and N. Goldman, Measuring the Chern number of Hofstadter bands with ultracold bosonic atoms, Nat. Phys. 11, 162 (2015).

[14] B. K. Stuhl, H.-I. Lu, L. M. Aycock, D. Genkina, and I. B. Spielman, Visualizing edge states with an atomic Bose gas in the quantum Hall regime, Science 349, 1514 (2015).

[15] M. E. Tai, A. Lukin, M. Rispoli, R. Schittko, T. Menke, D. Borgnia, P. M. Preiss, F. Grusdt, A. M. Kaufman, and M. Greiner, Microscopy of the interacting Harper-Hofstadter model in the few-body limit, arXiv:1612.05631.

[16] D. J. Thouless, M. Kohmoto, M. P. Nightingale, and M. den Nijs, Quantized Hall Conductance in a Two-Dimensional Periodic Potential, Phys. Rev. Lett. 49, 405 (1982).

[17] C. L. Kane and E. J. Mele, Quantum Spin Hall Effect in Graphene, Phys. Rev. Lett. 95, 226801 (2005).

[18] E. Zhao and A. Paramekanti, BCS-BEC Crossover on the Two-Dimensional Honeycomb Lattice, Phys. Rev. Lett. 97, 230404 (2006).

[19] N. B. Kopnin and E. B. Sonin, BCS Superconductivity of Dirac Electrons in Graphene Layers, Phys. Rev. Lett. 100, 246808 (2008).

[20] D. Jaksch, C. Bruder, J. I. Cirac, C. W. Gardiner, and P. Zoller, Cold Bosonic Atoms in Optical Lattices, Phys. Rev. Lett. 81, 3108 (1998); M. Greiner, O. Mandel, T. Esslinger, T. W. Hänsch, and I. Bloch, Quantum phase transition from a superfluid to a Mott insulator in a gas of ultracold atoms, Nature (London) 415, 39 (2002).

[21] R. O. Umucalılar and M. Ö. Oktel, Phase boundary of the boson Mott insulator in a rotating optical lattice, Phys. Rev. A 76, 055601 (2007); D. S. Goldbaum and E. J. Mueller, Vortex lattices of bosons in deep rotating optical lattices, Phys. Rev. A 77, 033629 (2008); A more exotic possibility is the presence of bosonic fractional quantum-Hall states, see, e.g., M. Hafezi, A. S. Sø rensen, E. Demler, and M. D. Lukin, Fractional quantum Hall effect in optical lattices, Phys. Rev. A 76, 023613 (2007); R. O. Umucalılar 
and E. J. Mueller, Fractional quantum Hall states in the vicinity of Mott plateaus, Phys. Rev. A 81, 053628 (2010).

[22] H. Zhai, R. O. Umucalılar, and M. Ö. Oktel, Pairing and Vortex Lattices for Interacting Fermions in Optical Lattices with a Large Magnetic Field, Phys. Rev. Lett. 104, 145301 (2010).

[23] M. Iskin, Attractive Hofstadter-Hubbard model with imbalanced chemical and vector potentials, Phys. Rev. A 91, 053606 (2015).

[24] R. O. Umucalılar and M. Iskin, Superfluid transition in the attractive Hofstadter-Hubbard model, Phys. Rev. A 94, 023611 (2016).

[25] S. Furukawa and M. Ueda, Global phase diagram of twocomponent Bose gases in antiparallel magnetic fields, Phys. Rev. A 90, 033602 (2014).

[26] D. Cocks, P. P. Orth, S. Rachel, M. Buchhold, K. Le Hur, and W. Hofstetter, Time-Reversal-Invariant HofstadterHubbard Model with Ultracold Fermions, Phys. Rev. Lett. 109, 205303 (2012).

[27] L. Wang, H.-H. Hung, and M. Troyer, Topological phase transition in the Hofstadter-Hubbard model, Phys. Rev. B 90, 205111 (2014).
[28] S. Peotta and P. Törmä, Superfluidity in topologically nontrivial flat bands, Nat. Commun. 6, 8944 (2015).

[29] T. Anzai and Y. Nishida, Two-dimensional Fermi gas in antiparallel magnetic fields, Phys. Rev. A 95, 051603 (2017).

[30] M. Kohmoto, Zero modes and the quantized Hall conductance of the two-dimensional lattice in a magnetic field, Phys. Rev. B 39, 11943 (1989).

[31] We also confirm this observation with the exact diagonalization of the mean-field Hamiltonian on a real-space lattice via the BdG prescription [23].

[32] See Supplemental Material at http://link.aps.org/ supplemental/10.1103/PhysRevLett.119.085301 for the dependence of the phase boundary on the temperature, particle filling and large $q$ values.

[33] M. P. A. Fisher, P. B. Weichman, G. Grinstein, and D. S. Fisher, Boson localization and the superfluid-insulator transition, Phys. Rev. B 40, 546 (1989).

[34] R. O. Umucalılar, H. Zhai, and M. Ö. Oktel, Trapped Fermi Gases in Rotating Optical Lattices: Realization and Detection of the Topological Hofstadter Insulator, Phys. Rev. Lett. 100, 070402 (2008). 